\title{
Black hole entropy and viscosity bound in Horndeski gravity
}

\author{
Xing-Hui Feng, ${ }^{a}$ Hai-Shan Liu, ${ }^{b, c}$ H. Lü̈ ${ }^{a}$ and C.N. Pope ${ }^{c, d}$ \\ ${ }^{a}$ Center for Advanced Quantum Studies, Department of Physics, \\ Beijing Normal University, Beijing 100875, China \\ ${ }^{b}$ Institute for Advanced Physics \& Mathematics, \\ Zhejiang University of Technology, Hangzhou 310023, China \\ ${ }^{c}$ George P. \& Cynthia Woods Mitchell Institute for Fundamental Physics and Astronomy, \\ Texas A\&M University, College Station, TX 7r843, U.S.A. \\ ${ }^{d}$ DAMTP, Centre for Mathematical Sciences, \\ Cambridge University, Wilberforce Road, Cambridge CB3 OWA, U.K. \\ E-mail: xhfengp@mail.bnu.edu.cn, hsliu.zju@gmail.com, \\ mrhonglu@gmail.com, pope@physics.tamu.edu
}

ABSTRACT: Horndeski gravities are theories of gravity coupled to a scalar field, in which the action contains an additional non-minimal quadratic coupling of the scalar, through its first derivative, to the Einstein tensor or the analogous higher-derivative tensors coming from the variation of Gauss-Bonnet or Lovelock terms. In this paper we study the thermodynamics of the static black hole solutions in $n$ dimensions, in the simplest case of a Horndeski coupling to the Einstein tensor. We apply the Wald formalism to calculate the entropy of the black holes, and show that there is an additional contribution over and above those that come from the standard Wald entropy formula. The extra contribution can be attributed to unusual features in the behaviour of the scalar field. We also show that a conventional regularisation to calculate the Euclidean action leads to an expression for the entropy that disagrees with the Wald results. This seems likely to be due to ambiguities in the subtraction procedure. We also calculate the viscosity in the dual CFT, and show that the viscosity/entropy ratio can violate the $\eta / S \geq 1 /(4 \pi)$ bound for appropriate choices of the parameters.

KEYwords: Black Holes, Classical Theories of Gravity, Holography and quark-gluon plasmas

ARXIV EPRINT: 1509.07142 


\section{Contents}

1 Introduction 1

2 Black holes in Horndeski gravity 3

2.1 The theory 3

2.2 Static black hole solutions 4

$\begin{array}{lll}2.3 & \text { Uniqueness of the Horndeski black hole solutions } & 7\end{array}$

3 Black hole entropy and thermodynamics $\quad 9$

$\begin{array}{llr}3.1 & \text { Wald entropy formula } & 9\end{array}$

$\begin{array}{lll}3.2 \text { Wald formalism } & 10\end{array}$

$\begin{array}{lll}3.3 & \text { Further comments on the entropy from Wald formalism } & 14\end{array}$

$\begin{array}{ll}3.4 & \text { Noether charge and mass of AdS planar black holes } \\ \end{array}$

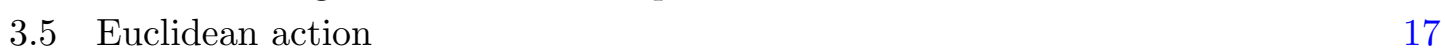

4 Viscosity/entropy ratio $\quad 19$

5 Conclusion $\quad 20$

\section{Introduction}

In the dictionary of gravity/gauge duality mappings in the AdS/CFT correspondence [1-3], perturbations of the metric are related to the energy-momentum tensor of the field theory in the boundary of the AdS spacetime [2-4]. In this picture, an AdS planar black hole is the gravitational dual of a certain ideal fluid. A widely valid relation between the shear viscosity and the entropy density was established, namely [5-8]

$$
\frac{\eta}{S}=\frac{1}{4 \pi}
$$

One way to understand this ratio is that it can be shown that the viscosity is proportional to the cross-section of the black hole for low-frequency massless scalar fields [8]. Alternatively, the shear viscosity is determined by the effective coupling constant of the transverse graviton on the horizon, by employing the membrane paradigm [9]. (This was confirmed by using the Kubo formula in $[10,11]$.) In [12], it was shown that the black hole entropy is determined by the effective Newtonian coupling at the horizon, and that it is thus not surprising that the ratio of the shear viscosity to the entropy density is universal, in the sense that the dependence of the quantities on the horizon is canceled. Recently, it was established that the relation (1.1) of the boundary theory is dual to a generalised Smarr relation obeyed by the bulk AdS planar black holes, thereby providing a new understanding of its universality, and its connection to the black hole thermodynamics [13]. There have 
been a number of papers in literature establishing the universality of the ratio (1.1) [14-17]. (See [18] for a review.)

The viscosity/entropy ratio (1.1) can, however, be violated when the bulk gravity theory is extended by the addition of higher-order curvature terms $[19,20] .{ }^{1}$ (See also, for further examples, [25-27].)

This leads us to one of the motivations for this paper, which is to investigate whether one can violate the ratio (1.1) without introducing higher-order curvature terms in the bulk theory. In a typical theory of Einstein gravity, matter fields couple to gravity minimally through the metric. A scalar field can also couple to gravity non-minimally, such as in Brans-Dicke theory [28], where the effective Newton constant varies in spacetime. However, it was established in [13] that the ratio (1.1) holds in general in such a theory. Scalar fields can, however, also couple non-minimally to gravity in other ways. In particular, their derivatives can couple to the curvature tensor. Horndeski considered a wide class of such gravity/scalar theories in the early seventies [29], focusing his attention on cases where the field equations, both for gravity and the scalar field, involve no higher than second derivatives. The Horndeski theories were rediscovered recently in studies of the covariantisation of Galileon theories [30].

The Horndeski terms take the form

$$
H^{(k)}=E_{\mu \nu}^{(k)} \partial^{\mu} \chi \partial^{\nu} \chi
$$

where the $E^{(k)}$ tensors are "energy-momentum tensors" associated with the Euler integrands of various order, namely

$$
E_{\mu}^{(k) \nu} \equiv \delta_{\mu \sigma_{1} \cdots \sigma_{2 k}}^{\nu \rho_{1} \cdots \rho_{2 k}} R_{\rho_{1} \rho_{2}}^{\sigma_{1} \sigma_{2}} \cdots R^{\sigma_{2 k-1} \sigma_{2 k}}{ }_{\rho_{2 k-1} \rho_{2 k}} .
$$

The $H^{(k)}$ terms are analogous to Euler integrands, in that they have the property that each field carries no more than a single derivative and hence the linearized equations of motion involve at most second derivatives. Thus although the theory involves higher-order derivatives, it contains no linear ghost excitations. In this paper, we shall consider Einstein gravity with a cosmological constant, together with just the two lowest-order Horndeski terms, namely

$$
H^{(0)}=g_{\mu \nu} \partial^{\mu} \chi \partial^{\mu} \chi, \quad H^{(1)}=-4 G_{\mu \nu} \partial^{\mu} \chi \partial^{\nu} \chi,
$$

where $G_{\mu \nu}$ is the Einstein tensor. We find that although the theory contains the curvature tensor only linearly, the viscosity/entropy ratio (1.1) no longer holds.

It is worth commenting that the viscosity can be computed by standard procedures using the AdS/CFT correspondence, involving the straightforward technique of studying linearised perturbations around the background bulk solution. The calculation of the viscosity/entropy ratio then hinges upon the proper definition of the entropy of the black hole. Since Hawking established the thermal radiation of a black hole [31, 32], there has been no ambiguity in establishing the black hole entropy in a generally-covariant theory.

\footnotetext{
${ }^{1}$ We shall not be concerned in this paper with other types of violation, due to the breaking of local rotational symmetry; see, for example, [21-24].
} 
In particular, in Einstein gravity minimally coupled to matter, the entropy is given by one quarter of the area of the horizon. This area law has been generalized to the Wald entropy formula when more complicated couplings or higher-order curvature terms are involved, namely [33, 34]

$$
S_{W}=-\frac{1}{8} \int_{+} d^{n-2} x \sqrt{h} \frac{\partial L}{\partial R^{a b c d}} \epsilon^{a b} \epsilon^{c d} .
$$

where $L$ is defined by the action $I=\int d^{n} x \sqrt{-g} L$. Applying this formula to static black holes with spherical, toric or hyperbolic isometries, the Horndeski terms (1.4) do not contribute to the Wald entropy $S_{W}$, and hence one might expect that the entropy would still be just one quarter of the horizon area. However, we find that this is in fact not the case. By examining the Wald procedure $[33,34]$ in detail, we find that in a theory such as Horndeski gravity there is an additional contribution to the entropy that is not encompassed by the usual Wald formula (1.5). It arises because the derivative of the scalar field diverges on the horizon in the black-hole solutions (although there is no physical divergence, since all invariants, such as $g^{\mu \nu} \partial_{\mu} \chi \partial_{\nu} \chi$, remain finite).

The paper is organised as follows. In section 2 we introduce the Horndeski theory that we shall be considering, and we review the static black hole solutions. These are known for all the cases of spherical, toroidal and hyperbolic horizon geometries. Our focus will be on the spherical and the toroidal horizons. We also include a demonstration of the uniqueness of the known static solutions. In section 3 we address the problem of calculating the entropy, and also the mass, of the static black holes. We begin by calculating the entropy using the standard Wald formula (1.5), and then we consider the application of the Wald formalism in more detail, showing that there is another contribution to the entropy that is not captured by (1.5). We show that in the case of the planar black holes (with toroidal horizons), the entropy expression we obtain is consistent with the computation of the Noether charge associated with a scaling symmetry of the black holes. We also consider the calculation of the Euclidean action, showing that, at least when following a naive regularisation procedure, this yields yet another result for the entropy, and the mass, that disagrees with those from the Wald formalism. In section 4 we calculate the shear viscosity in the dual boundary theory using the AdS/CFT correspondence, and hence we obtain an expression for the viscosity/entropy ratio. This is different from $1 /(4 \pi)$ on account of the Horndeski term, and we show that for an appropriate choice of the parameters it can violate the $\eta / S \geq 1 /(4 \pi)$ bound. The paper ends with conclusions in section 5 .

\section{Black holes in Horndeski gravity}

\subsection{The theory}

As we have discussed in the introduction, Horndeski gravity represents a class of higherderivative theories involving gravity with a non-minimally coupled scalar. The couplings differ from those in the Brans-Dicke theory, since in the Horndeski theories the scalar couples through its derivative to the curvature tensors. We shall focus on the Horndeski theory whose Lagrangian involves at most only linear curvature terms. As we shall show, 
the viscosity/entropy ratio (1.1) can be violated even in such a theory. The action is given by

$$
I=\frac{1}{16 \pi} \int d^{n} x \sqrt{-g} L, \quad L=\kappa(R-2 \Lambda)-\frac{1}{2}\left(\alpha g_{\mu \nu}-\gamma G_{\mu \nu}\right) \partial^{\mu} \chi \partial^{\nu} \chi,
$$

where $\kappa, \alpha$ and $\gamma$ are coupling constants, and $G_{\mu \nu} \equiv R_{\mu \nu}-\frac{1}{2} R g_{\mu \nu}$ is the Einstein tensor. Note that the theory is invariant under a constant shift of $\chi$. In a typical gravity theory with a scalar field, such as Brans-Dicke theory, one can define different metric frames by means of conformal scalings using the scalar field. However, for the Horndeski theory (2.1), this would lead to the breaking of the manifest constant shift symmetry of the scalar, and hence it would not be a natural field redefinition to make here.

The variation of the action (2.1) gives rise to

$$
\delta I=\frac{1}{16 \pi} \int d^{n} x \sqrt{-g}\left(E_{\mu \nu} \delta g^{\mu \nu}+E \delta \chi+\nabla_{\mu} J^{\mu}\right) .
$$

where

$$
\begin{aligned}
E_{\mu \nu}= & \kappa\left(G_{\mu \nu}+\Lambda g_{\mu \nu}\right)-\frac{1}{2} \alpha\left(\partial_{\mu} \chi \partial_{\nu} \chi-\frac{1}{2} g_{\mu \nu}(\partial \chi)^{2}\right)-\frac{1}{2} \gamma\left(\frac{1}{2} \partial_{\mu} \chi \partial_{\nu} \chi R-2 \partial_{\rho} \chi \partial_{(\mu} \chi R_{\nu)}{ }^{\rho}\right. \\
& -\partial_{\rho} \chi \partial_{\sigma} \chi R_{\mu}{ }^{\rho}{ }_{\nu}{ }^{\sigma}-\left(\nabla_{\mu} \nabla^{\rho} \chi\right)\left(\nabla_{\nu} \nabla_{\rho} \chi\right)+\left(\nabla_{\mu} \nabla_{\nu} \chi\right) \square \chi+\frac{1}{2} G_{\mu \nu}(\partial \chi)^{2} \\
& \left.-g_{\mu \nu}\left[-\frac{1}{2}\left(\nabla^{\rho} \nabla^{\sigma} \chi\right)\left(\nabla_{\rho} \nabla_{\sigma} \chi\right)+\frac{1}{2}(\square \chi)^{2}-\partial_{\rho} \chi \partial_{\sigma} \chi R^{\rho \sigma}\right]\right) \\
E= & \nabla_{\mu}\left(\left(\alpha g^{\mu \nu}-\gamma G^{\mu \nu}\right) \nabla_{\nu} \chi\right) .
\end{aligned}
$$

The total derivative term in (2.2) plays no role in the equations of motion

$$
E_{\mu \nu}=0, \quad E=0 .
$$

However, it does play an important role in the Wald formalism, which we shall present in section 3.2 .

\subsection{Static black hole solutions}

We now consider static black holes, with the ansatz

$$
d s_{n}^{2}=-h(r) d t^{2}+\frac{d r^{2}}{f(r)}+r^{2} d \Omega_{n-2, \epsilon}^{2}, \quad \chi=\chi(r),
$$

where $d \Omega_{n-2, \epsilon}^{2}$ with $\epsilon=1,0,-1$ is the metric for the unit $S^{n-2}$, the $(n-2)$-torus or the unit hyperbolic $(n-2)$-space. It is convenient to take $d \Omega_{n-2, \epsilon}^{2}=\bar{g}_{i j} d y^{i} d y^{j}$ for general values of $\epsilon$ to be the metric of constant curvature such that its Ricci tensor is given by $\bar{R}_{i j}=(n-3) \epsilon \bar{g}_{i j}$. We may, for example, take $d \Omega_{n-2, \epsilon}^{2}$ to be given by

$$
d \Omega_{n-2, \epsilon}^{2}=\frac{d u^{2}}{1-\epsilon u^{2}}+u^{2} d \Omega_{n-3}^{2},
$$

where $d \Omega_{n-3}^{2}$ is the metric of the unit $(n-3)$-sphere. 
It is clear from the equations of motion that $\chi=\chi_{0}$ (constant) is a solution, in which case, the Horndeski gravity reduces to Einstein gravity with a cosmological constant $\Lambda_{0}$. It follows that the Schwarzschild-AdS black hole is a solution of the theory. We shall regard this solution as being "trivial," in the sense of not yielding anything new. In addition, a one-parameter family of black hole solutions for which the scalar field is not a constant was constructed in [35]. (See also, [36, 37].) In this section, we would like to prove that these are the only black hole solutions from the ansatz (2.5) in which the scalar is $r$-dependent. First, we review the construction in [35].

The scalar equation of motion $E=0$ yields

$$
\left(r^{n-4} \sqrt{\frac{f}{h}}\left(\gamma\left((n-2) r f h^{\prime}+(n-2)(n-3)(f-\epsilon) h\right)-2 \alpha r^{2} h\right) \chi^{\prime}\right)^{\prime}=0
$$

There are two more equations that follow from $E_{\mu \nu}=0$ :

$$
\begin{aligned}
& \begin{array}{c}
4 \kappa\left((n-2) r f^{\prime}+(n-2)(n-3)(f-\epsilon)+2 \Lambda_{0} r^{2}\right)+2 \alpha r^{2} f \chi^{\prime 2} \\
+\gamma(n-2)\left(4 r f \chi^{\prime \prime}+\left(3 r f^{\prime}+(n-3)(f+\epsilon)\right) \chi^{\prime}\right) f \chi^{\prime}=0 \\
4 \kappa\left((n-2) r f h^{\prime}+(n-2)(n-3) h(f-\epsilon)+2 \Lambda r^{2} h\right)-2 \alpha r^{2} f h \chi^{\prime 2} \\
+\gamma(n-2)\left(3 r f h^{\prime}+(n-3)(3 f-\epsilon) h\right) f \chi^{\prime 2}=0
\end{array}
\end{aligned}
$$

In [35], a class of black hole solution was obtained by solving (2.7) by taking

$$
\gamma\left((n-2) r f h^{\prime}+(n-2)(n-3)(f-\epsilon) h\right)-2 \alpha r^{2} h=0 .
$$

(In other words, the integration constant in the first integral of (2.7) was taken to be zero, and $\chi^{\prime}$ was allowed to be non-zero, thus implying that its co-factor, given in (2.9), must be equal to zero.) This leads to the solution

$$
\begin{aligned}
h= & -\frac{\mu}{r^{n-3}}+\frac{8 \kappa\left[g^{2} r^{2}(2 \kappa+\beta \gamma)+2 \epsilon \kappa\right]}{(4 \kappa+\beta \gamma)^{2}} \\
& +\frac{(n-1)^{2} \beta^{2} \gamma^{2} g^{4} r^{4}}{\epsilon(n+1)(n-3)(4 \kappa+\beta \gamma)^{2}}{ }_{2} F_{1}\left[1, \frac{1}{2}(n+1) ; \frac{1}{2}(n+3) ;-\frac{n-1}{(n-3) \epsilon} g^{2} r^{2}\right], \\
f= & \frac{(4 \kappa+\beta \gamma)^{2}\left[(n-1) g^{2} r^{2}+(n-3) \epsilon\right]^{2}}{\left[(n-1)(4 \kappa+\beta \gamma) g^{2} r^{2}+4(n-3) \epsilon \kappa\right]^{2}} h, \quad \chi^{\prime 2}=\frac{\beta}{f}\left[1+\frac{(n-3) \epsilon}{(n-1) g^{2} r^{2}}\right]^{-1},
\end{aligned}
$$

which is valid for all values of $\epsilon$. In presenting the solution, we have introduced two parameters $(g, \beta)$ in place of the original parameters $(\alpha, \Lambda)$ in the Lagrangian, with

$$
\alpha=\frac{1}{2}(n-1)(n-2) g^{2} \gamma, \quad \Lambda=-\frac{1}{2}(n-1)(n-2) g^{2}\left(1+\frac{\beta \gamma}{2 \kappa}\right) .
$$

Note that the solution contains only one integration constant, $\mu$. All other parameters are those of the theory itself. Note also that since the dimension $n$ is an integer, the 
hypergeometric function reduces to polynomials with an arctan function in even dimensions, and with a log function in odd dimensions. To be explicit, we have

$$
\begin{aligned}
& n=\text { even : } \\
& { }_{2} F_{1}\left[1, \frac{1}{2}(n+1) ; \frac{1}{2}(n+3) ;-x\right]=\frac{(-1)^{n / 2}(n+1)}{x^{n / 2}}\left\{\frac{\arctan \sqrt{x}}{\sqrt{x}}-\left[\frac{\arctan \sqrt{x}}{\sqrt{x}}\right]_{\frac{n}{2}-1}\right\}, \\
& n=\text { odd : } \\
& { }_{2} F_{1}\left[1, \frac{1}{2}(n+1) ; \frac{1}{2}(n+3) ;-x\right]=\frac{(-1)^{\frac{n-1}{2}}(n+1)}{2 x^{\frac{n-1}{2}}}\left\{\frac{\log (1+x)}{x}-\left[\frac{\log (1+x)}{x}\right]_{\frac{n-3}{2}}\right\},
\end{aligned}
$$

where we use the notation $[F(x)]_{m}$ to denote the truncated power series expansion of $F(x)$ around $x=0$, in which only the terms up to and including $x^{m}$ are retained. Thus

$$
\left[\frac{\arctan \sqrt{x}}{\sqrt{x}}\right]_{\frac{n}{2}-1}=\sum_{p=0}^{n / 2-1} \frac{(-x)^{p}}{2 p+1}, \quad\left[\frac{\log (1+x)}{x}\right]_{\frac{n-3}{2}}=\sum_{p=0}^{\frac{n-3}{2}} \frac{(-x)^{p}}{p+1},
$$

for $n$ even and $n$ odd, respectively.

For static solutions of this kind, it is in fact always sufficient to construct the solution with $\epsilon=1$. The solutions for all other values of $\epsilon$, which we presented above, can then be obtained from the $\epsilon=1$ solution by means of the rescalings

$$
r \longrightarrow \frac{r}{\sqrt{\epsilon}}, \quad t \longrightarrow \sqrt{\epsilon} t, \quad d \Omega_{n-2}^{2} \longrightarrow \epsilon d \Omega_{n-2, \epsilon}^{2}, \quad \mu \longrightarrow \epsilon^{-(n-1) / 2} \mu
$$

From now on, we shall present results for the two specific cases $\epsilon=0$ and $\epsilon=1$.

$\boldsymbol{\epsilon}=\mathbf{0}$ solution: when $\epsilon=0$, the solution reduces to the very simple form

$$
h=f=g^{2} r^{2}-\frac{\mu}{r^{n-3}}, \quad \chi^{\prime 2}=\frac{\beta}{f} .
$$

Note that in this $\epsilon=0$ case, $\chi$ can be solved for explicitly, giving

$$
\chi=\frac{2 \sqrt{\beta}}{(n-1) g} \log \left(\sqrt{(g r)^{n-1}}+\sqrt{(g r)^{n-1}-\mu g^{n-3}}\right)+\chi_{0} .
$$

Thus the $\epsilon=0$ solution describes an AdS planar black hole, with the requirements that $\mu>0$ and $\beta \geq 0$. The horizon radius $r=r_{0}$ is given by $\mu=g^{2} r_{0}^{n-1}$. The Hawking temperature is given by

$$
T=\frac{(n-1) g^{2}}{4 \pi} r_{0}
$$

$\boldsymbol{\epsilon}=1$ solution: for $\epsilon=1$, the solution describes a spherically-symmetric and static black hole. In a large- $r$ expansion, if $n$ is even the functions $h$ and $f$ have the asymptotic forms

$$
\begin{aligned}
& h=g^{2} r^{2}-\frac{\mu}{r^{n-3}}+\sum_{k=0} \frac{c_{k}}{r^{2 k}}=g^{2} r^{2}+\frac{4 \kappa-\beta \gamma}{4 \kappa+\beta \gamma} \epsilon+\cdots, \\
& f=g^{2} r^{2}-\frac{\mu}{r^{n-3}}+\sum_{k=0} \frac{d_{k}}{r^{2 k}}=g^{2} r^{2}+\frac{4(n-1) \kappa+(n-5) \beta \gamma}{(n-1)(4 \kappa+\beta \gamma)} \epsilon+\cdots,
\end{aligned}
$$


where $\left(c_{k}, d_{k}\right)$ are constants, which are functions of the parameters $(\kappa, g, \beta)$ but independent of $\mu$. If $n$ is odd, then for $k=(n-3) / 2$, the quantity $c_{k}$ has an additional term proportional to $\log r$. This amounts to a logarithmically diverging addition to the mass coefficient $\mu$ at order $1 / r^{n-3}$. This in turn implies that $d_{k}$ has additional $\log r$ terms for all $k \geq(n-3) / 2$. Note that all the $\left(c_{k}, d_{k}\right)$ vanish for $\epsilon=0$.

The metric is asymptotic locally to AdS spacetime, and it cannot become pure AdS spacetime, regardless of the choice of the parameter $\mu$. To see that the solution describes a black hole, we note that $h$ is positive as $r$ goes to infinity, but becomes of order $-\mu / r^{n-3}$ as $r \rightarrow 0$, where there is a spacetime curvature singularity. Thus when $\mu>0$, there must exist some intermediate value of $r$, be an event horizon $r=r_{0}$, for which

$$
h\left(r_{0}\right)=0=f\left(r_{0}\right) .
$$

This implies that the parameter $\mu$ can be expressed in terms of the horizon radius $r_{0}$ in this $\epsilon=1$ case as

$$
\begin{aligned}
\mu=\frac{8 \kappa r_{0}^{n-3}}{(4 \kappa+\beta \gamma)^{2}}\left(2 \kappa+(2 \kappa+\beta \gamma) g^{2} r_{0}^{2}\right. & \\
& \left.\quad+\frac{(n-1)^{2} \beta^{2} \gamma^{2} g^{4} r_{0}^{4}}{8 \kappa(n-1)(n-3)}{ }_{2} F_{1}\left[1, \frac{1}{2}(n+1) ; \frac{1}{2}(n+3) ;-\frac{n-1}{(n-3)} g^{2} r_{0}^{2}\right]\right)
\end{aligned}
$$

Note that this relation between $\mu$ and $r_{0}$ is far more complicated than the simple expression $\mu=g^{2} r_{0}^{n-1}$ that holds in the $\epsilon=0$ case. The temperature of the $\epsilon=1$ black hole is given by

$$
T=\frac{\sqrt{h^{\prime}\left(r_{0}\right) f^{\prime}\left(r_{0}\right)}}{4 \pi}=\frac{(n-1) g^{2}}{4 \pi} r_{0}+\frac{(n-3) \kappa}{\pi(4 \kappa+\beta \gamma) r_{0}} .
$$

Note that if we set $\mu=0$, then the solution has no event horizon, and near $r=0$ the functions $h, f$ and $\chi$ have the forms

$$
\begin{aligned}
& h=\frac{16 \kappa^{2}}{(4 \kappa+\beta \gamma)^{2}}\left(1+\frac{(2 \kappa+\beta \gamma) g^{2} r^{2}}{2 \kappa}+\cdots\right), \\
& f=1+\frac{((n-3) \kappa-\beta \gamma) g^{2} r^{2}}{(n-3) \kappa}+\cdots \\
& \chi=\chi_{0}+\frac{(n-1) \beta}{2(n-3)} g r^{2}+\cdots
\end{aligned}
$$

Thus the $\mu=0$ solution is a smooth spherically-symmetric soliton, without any free parameters, that is asymptotic locally to AdS spacetime. There also exists a solution for $\epsilon=1$ in the limit of $4 \kappa+\beta \gamma=0$, but it does not describe a black hole.

\subsection{Uniqueness of the Horndeski black hole solutions}

We shall leave the discussion of the mass and entropy of the black holes to the next section. To close this section, we shall show that the solutions discussed above are in fact the only black holes with non-constant $\chi$ that are contained within the ansatz (2.5) in the theory. 
To show this, we return to the equation of motion (2.7) for the scalar field. One can immediately write down the first integral

$$
\chi^{\prime}=\frac{q r^{4-n} \sqrt{h / f}}{\gamma\left((n-2) r f h^{\prime}+(n-2)(n-3)(f-\epsilon) h\right)-2 \alpha r^{2} h},
$$

where $q$ is an integration constant. The solutions we discussed above were obtained by taking $q=0$. It was possible to find such solutions with $\chi^{\prime} \neq 0$ by imposing the relation (2.9), which in fact rendered the scalar equation of motion (2.7) trivial. If instead we take the integration constant $q$ to be non-zero, then $\chi^{\prime}$ is now determined by (2.24).

If a solution with $q \neq 0$ is to describe a black hole, there must be an event horizon at some radius $r=r_{0}$. The functions $h$ and $f$ near the horizon will have Taylor expansions of the form

$$
f=f_{1}\left(r-r_{0}\right)+f_{2}\left(r-r_{0}\right)^{2}+\cdots, \quad h=h_{1}\left(r-r_{0}\right)+h_{2}\left(r-r_{0}\right)^{2}+\cdots .
$$

It follows from (2.24) that $\chi^{\prime}$ near the horizon has the expansion

$$
\chi^{\prime}=\frac{\tilde{\chi}_{-1}}{r-r_{0}}+\tilde{\chi}_{0}+\tilde{\chi}_{1}\left(r-r_{0}\right)+\cdots .
$$

Substituting these expansions into the other equations of motion, we find that no such solutions can exist. In other words, the assumption that there exists a horizon, near which the expansions (2.25) would hold, is inconsistent with the equations of motion when $q \neq 0$. In order to have a solution with a horizon, we must therefore set $q=0$, which then reduces to the previous case discussed above. However, as mentioned already, in order for this solution not to be trivial, i.e. for $\chi^{\prime}$ to be non-vanishing, we must then also impose the condition (2.24). This leads to the black hole solution (2.10).

In the near-horizon region, the function $\chi$ in the black-hole solutions (2.10) has an expansion of the form

$$
\chi=\tilde{\chi}_{0}+\tilde{\chi}_{1}\left(r-r_{0}\right)^{\frac{1}{2}}+\tilde{\chi}_{2}\left(r-r_{0}\right)^{\frac{3}{2}}+\cdots .
$$

Substituting back into the equations of motion, we find that all the coefficients in the expansions can be expressed in terms of two parameters, $h_{1}$ and $r_{0}$. For example,

$$
f_{1}=\frac{(n-2)(n-3) \gamma \epsilon+2 \alpha r_{0}^{2}}{(n-2) \gamma r_{0}}, \quad \chi_{1}=\frac{2 \sqrt{(n-1) \beta} g r_{0}^{\frac{3}{2}}}{(n-1) g^{2} r_{0}^{2}+(n-3) \epsilon}, \quad \cdots .
$$

Thus the solution has three integration constants $\left(\tilde{\chi}_{0}, h_{1}, r_{0}\right)$. However, the parameters $\left(\tilde{\chi}_{0}, h_{1}\right)$ are trivial. It follows that the only non-trivial parameter is $r_{0}$, which is determined by $\mu$ in the final solution.

Finally we would like to emphasize again that $\beta$ is not an integration constant, but a parameter of the theory. For $\beta \neq 0$, there are two black holes, but each associated with a different vacuum. When $\beta=0$, there is only the Schwarzschild-AdS black hole solution in the theory. 


\section{Black hole entropy and thermodynamics}

In the previous section, we reviewed the Horndeski gravity theory, and its static black hole solutions. We identified the horizon and computed the temperature of these black holes. In this section, we consider various possible methods for calculating their entropy. It turns out that different well-established methods yield different answers. A correct answer of the entropy is important for studying the black hole thermodynamics, and it is paramount for determining the $\eta / S$ ratio, as we discussed in the introduction.

\subsection{Wald entropy formula}

First let us consider the well-known Wald entropy formula (1.5). It is straightforward to see that for the Horndeski Lagrangian $L$ given in (2.1), one has

$$
\begin{aligned}
T^{\mu \nu \rho \sigma} \equiv & \frac{\partial L}{\partial R_{\mu \nu \rho \sigma}} \\
= & \frac{1}{2} \kappa\left(g^{\mu \rho} g^{\nu \sigma}-g^{\nu \rho} g^{\mu \sigma}\right) \\
& +\frac{1}{8} \gamma\left[g^{\mu \rho} \chi^{\nu} \chi^{\sigma}-g^{\nu \rho} \chi^{\mu} \chi^{\sigma}+g^{\nu \sigma} \chi^{\mu} \chi^{\rho}-g^{\mu \sigma} \chi^{\nu} \chi^{\rho}-\left(g^{\mu \rho} g^{\nu \sigma}-g^{\nu \rho} g^{\mu \sigma}\right) \chi^{\lambda} \chi_{\lambda}\right],
\end{aligned}
$$

where we have defined $\chi_{\mu}=\partial_{\mu} \chi$. For the static black holes in the Horndeski theory, described in section 2, we find from (3.1) that the Wald entropy formula (1.5) for the entropy gives the same result as in standard Einstein gravity, namely one quarter of the area of the event horizon,

$$
S_{W}=\frac{1}{4} \kappa r_{0}^{n-2} \omega_{n-2},
$$

where $\omega_{n-2}$ is the volume of a unit $S^{n-2}$ in the $\epsilon=1$ case. For $\epsilon=0$, corresponding to a toroidal horizon, the periods of the circles forming the torus can be chosen arbitrarily, and we shall, for convenience, then take $\omega_{n-2}=1$ in this paper, and so correspondingly $S$ should then be viewed as the entropy density.

Since the static black hole solutions are characterised by only one parameter (i.e. one integration constant), it is guaranteed that one can obtain an expression for a "thermodynamic mass" by integrating the first law of black hole thermodynamics ${ }^{2}$

$$
d M=T d S .
$$

If we use the expression (3.2) for the entropy, then from the result for the Hawking temperature obtained in the previous section we therefore find

$$
\begin{aligned}
& \epsilon=0: \quad M=\frac{\kappa(n-2)}{16 \pi} \mu \\
& \epsilon=1: \quad M=\left(\frac{\kappa(n-2)}{16 \pi} g^{2} r_{0}^{n-1}+\frac{\kappa^{2}(n-2)}{4 \pi(4 \kappa+\beta \gamma)} r_{0}^{n-3}\right) \omega_{n-2} .
\end{aligned}
$$

\footnotetext{
${ }^{2}$ In a more general situation where there are further intensive/extensive pairs of thermodynamic variables contributing on the right-hand side of the first law for multi-parameter solutions, the integrability of the right-hand side can provide a non-trivial check on the correctness of the thermodynamic quantities. No such consistency check arises in the case of a one-parameter family of solutions, since all 1-forms are exact in one dimension.
} 
Note that in the $\epsilon=0$ case it was straightforward to express the mass in terms of the "mass parameter" $\mu$, because of the simple relation $\mu=g^{2} r_{0}^{n-1}$ for these planar black holes. On the other hand, the relation between $\mu$ and $r_{0}$ is much more complicated in the $\epsilon=1$ case, and is given in (2.21). Thus when $\epsilon=1$ the expression (3.5) for $M$ would become a complicated transcendental function of the mass parameter $\mu$.

On the face of it, the mass formula (3.4) for the $\epsilon=0$ case looks not unreasonable. In fact the thermodynamical quantities satisfy also the expected generalised Smarr relation

$$
M=\frac{n-2}{n-1} T S_{W}
$$

However, for the $\epsilon=1$ case, the mass formula (3.5) looks less reasonable. As mentioned above, it would be a complicated transcendental function of the "mass parameter" $\mu$. Whilst this fact, of itself, does not conclusively show that it must be incorrect, it does perhaps raise doubts about its likely validity, since it would be a very unusual kind of relation that is not normally seen in other black hole solutions. Furthermore, if the $\epsilon=1$ mass formula is called into question then this also raises questions about the validity of the $\epsilon=0$ mass formula.

In order to explore these issues in greater depth, we shall make a more detailed investigation of the Wald procedure, in order to see whether there are new subtleties that can arise in a theory such as that of Horndeski.

\subsection{Wald formalism}

Wald has developed a procedure for deriving the first law of thermodynamics by calculating the variation of a Hamiltonian derived from a conserved Noether current. The general procedure was presented in $[33,34]$. The Wald entropy formula (1.5) is a consequence of applying this procedure in rather generic higher-derivative theories. The Wald formalism has been used to study the first law of thermodynamics for asymptotically-AdS black holes in variety of theories, including Einstein-scalar [39, 40], Einstein-Proca [41], EinsteinYang-Mills [42], in gravities extended with quadratic-curvature invariants [43], and also for Lifshitz black holes [44]. However, the rather unusual-looking results that it led to for the mass of the $\epsilon=1$ black holes in section 3.1 raised the possibility that the formula (1.5) might not be valid for Horndeski gravity. For this reason, we shall now study in detail the application of the Wald formalism for the action (2.1).

A general variation of the fields in the action (2.1) was given in (2.2). The surface term $J^{\mu}$ is given by

$$
\begin{aligned}
J^{\mu} & =2 \frac{\partial L}{\partial R_{\rho \sigma \mu \nu}} \nabla_{\sigma} \delta g_{\rho \nu}-2 \nabla_{\nu} \frac{\partial L}{\partial R_{\rho \mu \nu \sigma}} \delta g_{\rho \sigma}+\frac{\partial L}{\partial\left(\nabla_{\mu} \chi\right)} \delta \chi \\
& =\left(\kappa J_{g}^{\mu}+\alpha J_{\chi}^{\mu}+\gamma\left(J_{g c}^{\mu}+J_{\chi c}^{\mu}\right)\right)
\end{aligned}
$$

with

$$
\begin{aligned}
& J_{g}^{\mu}=g^{\mu \rho} g^{\nu \sigma}\left(\nabla_{\sigma} \delta g_{\nu \rho}-\nabla_{\rho} \delta g_{\nu \sigma}\right), \quad J_{\chi}^{\mu}=-g^{\mu \nu} \nabla_{\nu} \chi \delta \chi, \quad J_{\chi c}^{\mu}=G^{\mu \nu} \nabla_{\nu} \chi \delta \chi \\
& J_{g c}^{\mu}=-\frac{1}{4}(\nabla \chi)^{2} J_{g}^{\mu}+\frac{1}{4} g^{\mu \rho} g^{\nu \sigma}\left[\nabla_{\sigma}(\nabla \chi)^{2} \delta g_{\nu \rho}-\nabla_{\rho}(\nabla \chi)^{2} \delta g_{\nu \sigma}\right]
\end{aligned}
$$




$$
\begin{aligned}
& +\frac{1}{2} g^{\mu \lambda} \nabla^{\rho} \chi \nabla^{\sigma} \chi \nabla_{\rho} \delta g_{\sigma \lambda}-\frac{1}{2} \nabla_{\rho}\left(\nabla^{\mu} \chi \nabla^{\sigma} \chi\right) g^{\rho \lambda} \delta g_{\sigma \lambda} \\
& -\frac{1}{4} g^{\mu \lambda} \nabla^{\rho} \chi \nabla^{\sigma} \chi \nabla_{\lambda} \delta g_{\rho \sigma}+\frac{1}{4} \nabla_{\lambda}\left(\nabla^{\rho} \chi \nabla^{\sigma} \chi\right) g^{\lambda \mu} \delta g_{\rho \sigma} \\
& -\frac{1}{4} g^{\rho \lambda} \nabla^{\mu} \chi \nabla^{\sigma} \chi \nabla_{\sigma} \delta g_{\rho \lambda}+\frac{1}{4} \nabla_{\sigma}\left(\nabla^{\sigma} \chi \nabla^{\mu} \chi\right) g^{\rho \lambda} \delta g_{\rho \lambda} .
\end{aligned}
$$

Following the Wald procedure, we can now define a 1-form $J_{(1)}=J_{\mu} d x^{\mu}$ and its Hodge dual

$$
\Theta_{(n-1)}=(-1)^{n+1} * J_{(1)}
$$

We now specialise to a variation that is induced by an infinitesimal diffeomorphism $\delta x^{\mu}=\xi^{\mu}$. One can show that

$$
J_{(n-1)} \equiv \Theta_{(n-1)}-i_{\xi} * L_{0}=-d * J_{(2)}
$$

after making use of the equations of motion. Here $i_{\xi}$ denotes a contraction of $\xi^{\mu}$ on the first index of the $n$-form $* L_{0}$. One can thus define an $(n-2)$-form $Q_{(n-2)} \equiv * J_{(2)}$, such that $J_{(n-1)}=d Q_{(n-2)}$. Note that we use the subscript notation " $(p)$ " to denote a $p$-form. To make contact with the first law of black hole thermodynamics, we take $\xi^{\mu}$ to be the time-like Killing vector that is null on the horizon. Wald shows that the variation of the Hamiltonian with respect to the integration constants of a specific solution is given by

$$
\delta \mathcal{H}=\frac{1}{16 \pi} \delta \int_{c} J_{(n-1)}-\frac{1}{16 \pi} \int_{c} d\left(i_{\xi} \Theta_{(n-1)}\right)=\frac{1}{16 \pi} \int_{\Sigma^{(n-2)}}\left(\delta Q_{(n-2)}-i_{\xi} \Theta_{(n-1)}\right)
$$

where $c$ denotes a Cauchy surface and $\Sigma^{(n-2)}$ is its boundary, which has two components, one at infinity and one on the horizon. Thus according to the Wald formalism, the first law of black hole thermodynamics is a consequence of

$$
\delta \mathcal{H}_{\infty}=\delta \mathcal{H}_{+} \cdot
$$

For the Horndeski gravity considered in this paper, we find

$$
\begin{aligned}
J_{\alpha_{1} \cdots \alpha_{n-1}}= & \text { E.O.M. }+2 \epsilon_{\alpha_{1} \cdots \alpha_{n-1} \mu} \nabla_{\nu}\left\{\kappa \nabla^{[\nu} \xi^{\mu]}-\frac{1}{4} \gamma(\nabla \chi)^{2} \nabla^{[\nu} \xi^{\mu]}+\frac{1}{2} \gamma \nabla^{[\nu}(\nabla \chi)^{2} \xi^{\mu]}\right. \\
& \left.+\frac{1}{2} \gamma \nabla^{\sigma} \chi \nabla^{[\nu} \chi \nabla_{\sigma} \xi^{\mu]}-\frac{1}{2} \gamma \nabla_{\sigma}\left(\nabla^{\sigma} \chi \nabla^{[\nu} \chi\right) \xi^{\mu]}-\frac{1}{2} \gamma \nabla^{[\nu}\left(\nabla^{\mu]} \chi \nabla^{\sigma} \chi\right) \xi_{\sigma}\right\}, \\
Q_{\alpha_{1} \cdots \alpha_{n-2}}= & \epsilon_{\alpha_{1} \cdots \alpha_{n-2} \mu \nu}\left\{\frac{\partial L}{\partial R_{\mu \nu \rho \sigma}} \nabla_{\rho} \xi_{\sigma}-2 \xi_{[\sigma} \nabla_{\rho]}\left(\frac{\partial L}{\partial R_{\mu \nu \rho \sigma}}\right)\right\} \\
= & \epsilon_{i_{1} \cdots i_{n-2} \mu \nu}\left\{\kappa \nabla^{\mu} \xi^{\nu}-\frac{1}{4} \gamma(\nabla \chi)^{2} \nabla^{\mu} \xi^{\nu}+\frac{1}{2} \gamma \nabla^{\sigma} \chi \nabla^{\mu} \chi \nabla_{\sigma} \xi^{\nu}\right. \\
& \left.+\frac{1}{2} \gamma\left(\nabla^{\mu}(\partial \chi)^{2}\right) \xi^{\nu}-\frac{1}{2} \gamma \nabla_{\sigma}\left(\nabla^{\sigma} \chi \nabla^{\mu} \chi\right) \xi^{\nu}-\frac{1}{2} \gamma \nabla^{\mu}\left(\nabla^{\nu} \chi \nabla^{\sigma} \chi\right) \xi_{\sigma}\right\}, \\
\left(i_{\xi} \Theta\right)_{\alpha_{1} \cdots \alpha_{n-2}}= & \epsilon_{\alpha_{1} \cdots \alpha_{n-2} \mu \lambda}\left(2 \frac{\partial L}{\partial R_{\rho \sigma \mu \nu}} \nabla_{\sigma} \delta g_{\rho \nu}-2 \nabla_{\nu} \frac{\partial L}{\partial R_{\rho \mu \nu \sigma}} \delta g_{\rho \sigma}+\frac{\partial L}{\partial\left(\nabla_{\mu} \chi\right)} \delta \chi\right) \xi^{\lambda} .
\end{aligned}
$$


To specialise to our static black hole ansatz (2.5), the result for the Lagrangian with $\gamma=0$ is well established (see, for example, $[39,40]$ ), and is given by

$$
\begin{aligned}
Q_{\kappa, \alpha} & =r^{n-2} \kappa \sqrt{\frac{f}{h}} h^{\prime} \Omega_{(n-2)}, \\
i_{\xi} \Theta_{\kappa, \alpha} & =-r^{n-2} \sqrt{\frac{h}{f}}\left(\kappa\left(-\frac{f}{h} \delta h^{\prime}+\frac{f h^{\prime}}{2 h^{2}} \delta h-\frac{h^{\prime}}{2 h} \delta f-\frac{n-2}{r} \delta f\right)-\alpha f \chi^{\prime} \delta \chi\right) \Omega_{(n-2)}, \\
\left(\delta Q-i_{\xi} \Theta\right)_{\kappa, \alpha} & =-r^{n-2} \sqrt{\frac{h}{f}}\left(\kappa \frac{n-2}{r} \delta f+\alpha f \chi^{\prime} \delta \chi\right) \Omega_{(n-2)},
\end{aligned}
$$

We find that the contributions associated with the $\gamma$ term in the action are given by

$$
\begin{aligned}
Q_{\gamma} & =-\frac{1}{2}(n-2) \gamma r^{n-3} \sqrt{\frac{h}{f}} f^{2} \chi^{\prime 2} \Omega_{(n-2)}, \\
i_{\xi} \Theta_{\gamma} & =-\frac{1}{2}(n-2) \gamma r^{n-3} \sqrt{\frac{h}{f}} f^{2}\left(\chi^{\prime 2} \frac{\delta h}{2 h}+\left(\frac{n-3}{r}\left(1-\frac{\epsilon}{f}\right)+\frac{h^{\prime}}{h}\right) \chi^{\prime} \delta \chi\right) \Omega_{(n-2)}, \\
\left(\delta Q-i_{\xi} \Theta\right)_{\gamma} & =\frac{1}{2}(n-2) \gamma r^{n-3} \sqrt{\frac{h}{f}} f^{2}\left(-\frac{3}{2} \chi^{\prime 2} \frac{\delta f}{f}-\delta\left(\chi^{\prime 2}\right)\right. \\
& \left.+\left(\frac{n-3}{r}\left(1-\frac{\epsilon}{f}\right)+\frac{h^{\prime}}{h}\right) \chi^{\prime} \delta \chi\right) \Omega_{(n-2)},
\end{aligned}
$$

We now apply the Wald formalism to the black hole solutions. First, we note that as a consequence of equation (2.9), when we add the contributions in (3.14) and (3.15) the $\chi^{\prime} \delta \chi$ in the total expression cancel, giving the result

$$
\delta Q-i_{\xi} \Theta=-(n-2) r^{n-3} \sqrt{\frac{h}{f}}\left[\left(\kappa-\frac{3}{4} \gamma f \chi^{\prime 2}\right) \delta f+\gamma f^{2} \delta\left(\chi^{\prime 2}\right)\right] .
$$

In fact, as can be seen from the expression for $\chi^{\prime 2}$ in for the black hole solutions in (2.10), we have $\delta\left(f \chi^{\prime 2}\right)=0$, and so (3.16) can be further simplified, to give

$$
\delta Q-i_{\xi} \Theta=-(n-2) r^{n-3} \sqrt{\frac{h}{f}}\left(\kappa+\frac{1}{4} \gamma f \chi^{\prime 2}\right) \delta f .
$$

We first consider the simpler case of the $\epsilon=0$ AdS planar black holes, for which $f \chi^{\prime 2}=\beta$. We find

$$
\begin{aligned}
\delta \mathcal{H}_{\infty} & =\frac{(n-2) \kappa}{16 \pi}\left(1+\frac{\beta \gamma}{4 \kappa}\right) \delta \mu, \\
\delta \mathcal{H}_{+} & =\frac{(n-1)(n-2) g^{2} \kappa}{16 \pi}\left(1+\frac{\beta \gamma}{4 \kappa}\right) r_{0}^{n-2} \delta r_{0} .
\end{aligned}
$$

Thus we see indeed that $\delta H_{\infty}=\delta H_{+}$, since $\mu=g^{2} r_{0}^{n-1}$. This implies that we can define the mass and entropy as

$$
M=\frac{(n-2) \kappa}{16 \pi}\left(1+\frac{\beta \gamma}{4 \kappa}\right) \mu, \quad S=\frac{1}{4} \kappa\left(1+\frac{\beta \gamma}{4 \kappa}\right) r_{0}^{n-2},
$$


such that

$$
\delta H_{\infty}=\delta M, \quad \delta H_{+}=T \delta S .
$$

The first law of black hole thermodynamics (3.3) then follows straightforwardly from the Wald identity (3.12). However the factor $1+\beta \gamma /(4 \kappa)$ in both the entropy and the mass disagrees with the results in (3.2) and (3.4) that we obtained in section 3.1 from a direct application of the Wald entropy formula (1.5) and the integration of the first law $d M=T d S$.

The case of the spherically-symmetric black holes with $(\epsilon=1)$ is more complicated. We find that $\delta \mathcal{H}$ evaluated on the horizon takes the general form

$$
\delta \mathcal{H}_{+}=\frac{(n-2) \omega_{n-2} T}{64}\left(16 \kappa+\gamma f_{1} \tilde{\chi}_{1}^{2}\right) r_{0}^{n-3} \delta r_{0},
$$

where $f_{1}$ and $\tilde{\chi}_{1}$ are coefficients in the near-horizon expansions defined in (2.25) and (2.27). For our specific $\epsilon=1$ solution, we have

$$
\begin{aligned}
f_{1} & =(n-1) g^{2} r_{0}+\frac{n-3}{r_{0}}, \quad \tilde{\chi}_{1}=\frac{2 \sqrt{(n-1) \beta} g r_{0}^{\frac{3}{2}}}{(n-1) g^{2} r_{0}^{2}+n-3}, \\
h_{1} & =\frac{\left((n-1)(4 \kappa+\beta \gamma) g^{2} r_{0}^{2}+4(n-3) \kappa\right)^{2}}{(4 \kappa+\beta \gamma)\left((n-2) g^{2} r_{0}^{2}+n-3\right) r_{0}} .
\end{aligned}
$$

Thus if we define $\delta \mathcal{H}_{+}=T d S$, with $T$ given in (2.22), we find that the entropy is given by

$$
\begin{aligned}
S=\omega_{n-2}\left[\frac{1}{4} \kappa r_{0}^{n-2}\right. & +\frac{(n-1)(n-2) \beta \gamma g^{2} r_{0}^{n}}{16 n(n+2)(n-3)^{2}}((n+2)(n-3) \\
& \left.\left.-n(n-1) g^{2} r_{0}^{2} F_{1}\left[1, \frac{1}{2}(n+2) ; \frac{1}{2}(n+4) ;-\frac{n-1}{n-3} g^{2} r_{0}^{2}\right]\right)\right] .
\end{aligned}
$$

Note that the first term inside the square brackets gives precisely the result we saw earlier (3.2) for Wald entropy $S_{W}$, derived using the formula (1.5). The remaining contribution in the square brackets is proportional to $\gamma$, the coefficient of the Horndeski term in the action (2.1).

To derive the first law, we evaluate the $\delta \mathcal{H}$ at asymptotic infinity, and we find

$$
\delta H_{\infty}=\frac{(n-2) \kappa \omega_{n-2}}{16 \pi}\left(1+\frac{\beta \gamma}{4 \kappa}\right) \delta \mu
$$

This implies that the mass is given by

$$
M=\frac{(n-2) \kappa \omega_{n-2}}{16 \pi}\left(1+\frac{\beta \gamma}{4 \kappa}\right) \mu .
$$

This turns out to be exactly the same form as that in the $\epsilon=0$ AdS planar black hole. It is now straightforward to verify that the first law (3.3) is indeed satisfied. Note that $\chi_{0}$, being a constant shift integration constant of $\chi$, plays no role in the first law.

It is worth commenting that for the $\epsilon=0$ solutions, the masses we obtained in (3.4) and in (3.19) by the two different methods are both proportional to $\mu$. The only difference is in the constant prefactor coefficient. This on its own makes it difficult to judge which 
is the more reasonable result. However, when $\epsilon=1$, the difference becomes more striking. The result (3.25) from the detailed Wald procedure that we presented in this paper is seemingly more plausible, for two reasons. Firstly, the mass is simply proportional to the parameter $\mu$, instead of being a convoluted transcendental function of $\mu$. Secondly, the mass dependence on $\mu$ is the same for both the $\epsilon=0$ and $\epsilon=1$ solution. In solutions with no additional scalar hair, and since the $\epsilon=0$ solution can be obtained as a scaling limit of the $\epsilon=1$ solution, this conclusion would seem to be reasonable.

\subsection{Further comments on the entropy from Wald formalism}

Having derived the first law of thermodynamics and also the entropy in section 3.2, using the general Wald formalism, we now examine the somewhat unusual features of the black holes in Horndeski gravity that lead to the breakdown of the standard Wald entropy formula (1.5). It follows from (3.13) that for the static ansatz (2.5) that

$$
Q_{(n-2)}=2 h^{\prime} \sqrt{\frac{f}{h}} S_{(n-2)}^{0 \hat{0} \hat{0} \hat{1}}-4 h T_{; 1}^{0101} \Omega_{(n-2)},
$$

where the hatted indices are tangent-space indices, the semicolon denotes a covariant derivative and

$$
T^{\mu \nu \rho \sigma} \equiv \frac{\partial L}{\partial R_{\mu \nu \rho \sigma}}, \quad S_{(n-2)}^{0 \hat{0} \hat{0} \hat{1}}=T^{\hat{0} \hat{1} \hat{1} \hat{1}} r^{n-2} \Omega_{(n-2)} .
$$

Note that 0 is the time direction and 1 is the $r$ direction. The expression for $T^{\mu \nu \rho \sigma}$ for the Horndeski gravity is given by (3.1). Typically, one evaluates $Q_{(n-2)}$ on the horizon at $r=r_{0}$, with $h=h_{1}\left(r-r_{0}\right)+\cdots$ and $f=f_{1}\left(r-r_{0}\right)+\cdots$, and so the second term on the right-hand side of (3.26) vanishes and hence, as was observed in [33, 34], we find

$$
\frac{1}{16 \pi} \int_{r=r_{0}} Q_{(n-2)}=T S_{W}
$$

where $S_{W}$ is the standard Wald entropy, given by (1.5).

Establishing the variational identity (3.12) is more subtle, even for the standard case of Einstein gravity. It requires that we evaluate $\delta Q$ on the horizon. Naively, one would simply obtain $\delta T S_{W}+T \delta S_{W}$ from (3.28), and then one would expect that the $\delta T S_{W}$ term would be cancelled by the $i_{\xi} \Theta$ contribution in (3.11), leading to

$$
\delta \mathcal{H}_{+}=T \delta S_{W} .
$$

However, in order to evaluate the variation properly, we need to expand (3.28) up to order $\left(r-r_{0}\right)$, since $\delta\left(r-r_{0}\right)=-\delta r_{0}$ and so it is non-zero even in the limit when one sets $r=r_{0}$ on the horizon. The net effect is that all the terms in $\delta Q_{(n-2)}$ are cancelled out by terms in $i_{\xi} \Theta$, and in fact the $T \delta S$ term arises from the remaining terms in $i_{\xi} \Theta$ alone.

To be specific, let us examine $\delta Q-i_{\xi} \Theta$ for a spherically-symmetric black hole in pure Einstein gravity coupled to a massless scalar, as given by (3.14). If we first perform Taylor expansions of $Q$ and $i_{\xi} \Theta$, as given in the first two equations in (3.14), around the horizon at $r=r_{0}$, then indeed the above statement can be verified. The final equation in (3.14) gives an alternative but equivalent evaluation with the variation $\delta Q$, which makes the 
observation more apparent. We may evaluate $\delta Q$ first, and then set $r=r_{0}$. In this case, the $r^{n-2}$ factor in $Q_{\kappa, \alpha}$ just depends on the coordinate $r$, and hence is not varied. With this procedure, we find that all the terms in $\delta Q_{\kappa, \alpha}$ are cancelled out by terms in $i_{\xi} \Theta_{\kappa, \alpha}$, leading to the third equation of (3.14). Thus using this procedure, we find that the $\delta \mathcal{H}_{+}=T \delta S$ term for the usual Einstein gravity arises from the $(n-2) \delta f / r$ term in $i_{\xi} \Theta$ in (3.14). This term corresponds to

$$
r^{n-2} \sqrt{\frac{h}{f}} \frac{2}{r f} g_{i j} T^{1 i 1 j} \delta f \Omega_{(n-2)} .
$$

It is rather intriguing how this term is ultimately related to $S_{W}$ which involves only $T^{0101}$. Indeed, we see from (3.1) that in vielbein components, $T^{\hat{0} \hat{1} \hat{0} \hat{1}}=-\frac{1}{2} \kappa$ and $T^{\hat{1} \hat{i} \hat{1} \hat{j}}=\frac{1}{2} \kappa \delta^{i j}$ for the Horndeski black hole solutions. In particular, the $\gamma$ term does not contribute in either case.

In the black holes of Horndeski gravity there are further subtleties. Firstly, the $\alpha$ term in $i_{\xi} \Theta_{\kappa, \alpha}$ in (3.14) does not vanish for these solutions, and can contribute a term to the entropy that is not contained in $S_{W}$. Furthermore, although the second term in (3.26) vanishes on the horizon, its variation does not. This extra term can be seen in the form of $Q_{\gamma}$ in (3.15). Thus $\left(\delta Q-i_{\xi} \Theta\right)_{\gamma}$ in (3.15) will give an additional contribution to the entropy that is over and above that of the standard Wald contribution $S_{W}$. Thus we now have

$$
\delta \mathcal{H}_{+}=T \delta S, \quad \text { with } \quad S \neq S_{W} .
$$

However, the Wald identity (3.12), as we have seen, continues to hold. The non-vanishing contributions from both the $\alpha$ and the $\gamma$ terms have the same essential origin, namely that the scalar field $\chi$ is not regular on the horizon, but rather, it has a branch cut singularity, as shown in (2.27).

One might question whether this is compatible with the interpretation of the solutions as black holes. However, as we have remarked in section 2.1, the scalar $\chi$ in Horndeski gravity is like an axion, in the sense that it enters the theory only through its derivative. In particular, therefore, it would not be natural to define different conformally-scaled metric frames (in the manner that one does with the dilaton in string theory), since that would break the manifest axionic shift symmetry of $\chi$. Furthermore, all invariant polynomials constructed from $\partial_{\mu} \chi$ with the metric and the Riemann tensor are regular on the horizon. For example, $g^{\mu \nu} \partial_{\mu} \chi \partial_{\nu} \chi$ is finite and non-zero on the horizon. (These properties can be seen from the fact that the vielbein components of the gradient of $\chi$ are finite everywhere, including on the horizon, since one just has $E_{\hat{1}}^{\mu} \partial_{\mu} \chi=\sqrt{f} \chi^{\prime}=\sqrt{\beta}\left[1-(n-3) \epsilon /\left((n-2) g^{2} r^{2}\right)\right]^{-1 / 2}$, with all other components vanishing, where $E_{\hat{a}}^{\mu}$ is the inverse vielbein.) This supports the idea that these solutions admit a valid black hole interpretation, but at the price that the Wald entropy formula (1.5) no longer provides the complete expression for the entropy. However, the identity (3.12), and hence the first law of black hole thermodynamics, continues to hold, with the entropy being derived from the strict application of the Wald formalism. 


\subsection{Noether charge and mass of AdS planar black holes}

In the previous subsections, we described two different methods for calculating the entropy and mass of the Horndeski black holes, one based on the use of the Wald formula (1.5) for the entropy, and the other based on a more detailed consideration of the Wald formalism. In both these approaches, we did not use independent procedures to calculate the mass and entropy, but rather, we relied on the use of the first law of thermodynamics to obtain one from the other. Since the black-hole solutions are characterised by only one parameter, there is no non-trivial integrability check, in the sense that the right-hand side of the first law $d M=T d S$ would be integrable regardless of whether the expression for the entropy was correct or not. The fact that the two approaches led to different results calls for an independent check on the calculation of the mass, or the entropy. Even though the mass and entropy obtained from the Wald formalism in section 3.2 seems to be more reasonable, the mass is determined through an integration of the first law, rather than directly, in this case. A question one can ask is whether the mass is indeed a conserved quantity.

For the AdS planar black holes (i.e. the $\epsilon=0$ solutions), this question can be answered by means of a simple Noether calculation. For $\epsilon=0$, we rewrite the ansatz as

$$
d s^{2}=d \rho^{2}-a(\rho)^{2} d t^{2}+b(\rho)^{2} d \Omega_{\epsilon}^{2}, \quad \chi=\chi(\rho) .
$$

The effective one-dimensional Lagrangian becomes

$$
\begin{aligned}
L & =\frac{1}{16 \pi} a b^{n-2}\left(\kappa\left(R-2 \Lambda_{0}\right)-\frac{1}{2} \alpha \chi^{\prime 2}+\frac{1}{2} \gamma G_{11} \chi^{\prime 2}\right), \\
R & =-\frac{2 a^{\prime \prime}}{a}-\frac{2(n-2) b^{\prime \prime}}{b}-\frac{2(n-2) a^{\prime} b^{\prime}}{a b}-\frac{(n-2)(n-3) b^{\prime 2}}{b^{2}}+\frac{\epsilon(n-2)(n-3)}{b^{2}}, \\
G_{11} & =\frac{(n-2) a^{\prime} b^{\prime}}{a b}+\frac{(n-2)(n-3) b^{\prime 2}}{2 b^{2}}-\frac{\epsilon(n-2)(n-3)}{2 b^{2}} .
\end{aligned}
$$

where a prime denotes a derivative with respect to $\rho$. The Lagrangian is invariant under the global scaling

$$
a \rightarrow \lambda^{2-n} a, \quad b \rightarrow \lambda b .
$$

This global symmetry yields a conserved Noether charge

$$
\mathcal{Q}_{N}=\frac{1}{16 \pi}(n-2) b^{n-3}\left(b a^{\prime}-a b^{\prime}\right)\left(4 \kappa+\gamma \chi^{\prime 2}\right) .
$$

In terms of the coordinates of the original ansatz (2.5), we have

$$
\mathcal{Q}_{N}=\frac{n-2}{32 \pi} r^{n-3} \sqrt{\frac{f}{h}}\left(r h^{\prime}-2 h\right)\left(4 \kappa+\gamma f \chi^{\prime 2}\right) .
$$

Substituting the AdS planar black hole solution into this Noether charge formula, we find

$$
\mathcal{Q}_{N}=\frac{(n-1)(n-2) \kappa}{8 \pi}\left(1+\frac{\beta \gamma}{4 \kappa}\right) \mu=2(n-1) M .
$$

Thus we see that $\mathcal{Q}_{N}$ is the same as the mass obtained from the Wald formalism in section 3.2 , up to some purely numerical constants. This supports the conclusion that the mass and entropy obtained in section 3.2 are valid, whilst the results in section 3.1 are not. 


\subsection{Euclidean action}

An alternative method that has been used for calculating thermodynamic quantities for black hole solutions is by means of the quantum statistical relation

$$
\Phi_{\text {thermo }} \equiv M-T S=I T
$$

first proposed for quantum gravity in [38] . Here $\Phi_{\text {thermo }}$ denotes the thermodynamic potential, or the free energy, and $I$ is the Euclidean action. The regularised Euclidean action was calculated for the $\epsilon=1$ Horndeski black hole in four dimensions in [35]. We have repeated that calculation, and obtained the same result (save for an overall factor of 2 discrepancy). However, the resulting expressions for mass and entropy are quite different from those in sections 3.1 or 3.2 , and are given by

$$
\begin{aligned}
M & =\frac{1}{2} \kappa\left(1+\frac{\beta \gamma}{4 \kappa}\right) \mu-\frac{3 \beta \gamma g^{2} r_{0}^{3}\left(4 \kappa\left(3 g^{2} r_{0}^{2}+1\right)+3 \beta \gamma g^{2} r_{0}^{2}\right)}{8(4 \kappa+\beta \gamma)\left(1+3 g^{2} r_{0}^{2}\right)\left(4 \kappa\left(3 g^{2} r_{0}^{2}-1\right)+3 \beta \gamma g^{2} r_{0}^{2}\right)} \\
S & =\kappa \pi r_{0}^{2}+\frac{3 \pi \beta \gamma g^{2} r_{0}^{4}\left(4 \kappa+3(4 \kappa+\beta \gamma) g^{2} r_{0}^{2}\right)}{2\left(1+3 g^{2} r_{0}^{2}\right)\left(4 \kappa-3(4 \kappa+\beta \gamma) g^{2} r_{0}^{2}\right)}
\end{aligned}
$$

Note that when $\beta=0$, for which the black hole reduces to the standard Schwarzschild-AdS one, we get $M=\frac{1}{2} \mu$ and $S=\kappa \pi r_{0}^{2}$, as one would expect. It is clear that the mass suffers from the same shortcoming as the one we obtained from the Wald entropy formula in (3.5), in that it becomes a convoluted transcendental function of $\mu$ for non-vanishing $\beta$. (It is a different transcendental function from the one following from (3.5), however.)

The calculation for the $\epsilon=0$ AdS planar black holes (2.16) is much easier, and can be straightforwardly carried out for a general spacetime dimension $n$. The regularised Euclidean action can be defined by subtracting the action of the background $\mu=0$ vacuum from the action for the black hole itself, namely

$$
I_{r e g}=I_{E}\left[g_{\mu \nu}, \chi\right]-I_{E}\left[g_{\mu \nu}^{(0)}, \chi^{(0)}\right],
$$

where $g_{\mu \nu}^{(0)}$ and $\chi^{(0)}$ are the background field obtained by setting $\mu=0$ in the black hole solution (2.16). We find

$$
I_{\text {reg }}=-\frac{\kappa}{16(n-1)}\left(1-\frac{(n-2) \beta \gamma}{4 \kappa}\right) r_{0}^{n-2} .
$$

Note that in this calculation, we have set $\omega_{n-2}=1$, so that the resulting extensive quantities are densities. Using the quantum statistical relation (3.38) and the thermodynamic first law (3.3), we then find that the free energy, mass, temperature and entropy for the $\epsilon=0$ black holes are given by

$$
\begin{aligned}
F & =-\frac{\kappa \mu}{16 \pi}\left(1-\frac{(n-2) \beta \gamma}{4 \kappa}\right), & M & =-(n-2) F \\
T & =\frac{g^{2}(n-1) r_{0}}{4 \pi}, & S & =\frac{1}{4} \kappa r_{0}^{n-2}-\frac{1}{16}(n-2) \beta \gamma r_{0}^{n-2} .
\end{aligned}
$$


These expressions also disagree, in this case by constant overall factors, with the $\epsilon=0$ results obtained in sections 3.1 and 3.2. Taken in isolation, it would be hard to make any judgment as to whether these expressions were trustworthy or not. Interestingly the generalized Smarr relation (3.6) is also satisfied. However, the $\epsilon=1$ results (3.39) for the mass and the entropy certainly raise questions about the validity of this calculation using the Euclidean action.

There is another method that has been used in order to obtain a finite Euclidean action, by adding a surface term and a counterterm. Taking $n=4$ dimensions as an example, the whole action is then given by

$$
I=I_{b u l k}-2 I_{\mathrm{GH}}-I_{\mathrm{ct}},
$$

where $I_{\mathrm{GH}}$ is the standard Gibbons-Hawking surface term, and for $\epsilon=0$, the counterterm is given by

$$
I_{\mathrm{ct}}=\kappa \int d x^{3} \sqrt{\gamma} c_{1} g, \quad \text { with } \quad c_{1}=4+\frac{\beta \gamma}{\kappa},
$$

The $\gamma$ in the square root is the determinant of induced metric $\gamma_{\mu \nu}$. With these combinations, the total action is the same as the result of regularization. For $\epsilon=1$, the counterterm is

$$
I_{\mathrm{ct}}=\kappa \int d x^{3} \sqrt{\gamma}\left(c_{1} g+\frac{c_{2} R[\gamma]}{g}\right), \quad \text { with } \quad c_{1}=4+\frac{\beta \gamma}{\kappa}, \quad c_{2}=1-\frac{\beta \gamma}{4 \kappa}
$$

and the value of the action has an additional term linear the imaginary-time period (i.e. inversely proportional to the temperature), in comparison to that of the regularized calculation above:

$$
I_{\text {renorm }}=I_{\text {reg }}+\frac{\sqrt{3} \pi \beta^{2} \gamma^{2}}{12 g(4 \kappa+\beta \gamma)} \frac{\epsilon}{T} .
$$

The effect on the thermodynamics is that the entropy is unchanged, but the mass acquires an additive contribution in the spherically-symmetric $\epsilon=1$ solutions, independent of the parameter in the solutions. This is not surprising, since when $\epsilon=1$, the $\mu=0$ solution is not vacuum AdS spacetime, but instead a smooth soliton, which has a constant mass. In the earlier regularisation by subtracting the background, this constant energy was subtracted out.

The question remains as to how one might reconcile the results for the entropy and the mass, as calculated from the regularised Euclidean action, with our previous, and different, results obtained using the Wald formalism. We do not have a definitive resolution to this puzzle, other than to suggest that because of the rather unusual features of the blackhole solutions in Horndeski gravity, it may be that the naive application of a subtraction procedure to obtain a regularised Euclidean action may be inherently ambiguous. In a somewhat related context, it was found in [45] that attempts to employ the Abbott-Deser method [46] to calculate the mass of asymptotically-AdS black holes foundered on ambiguities in the subtraction procedure in some cases, for solutions in gauged supergravities where scalar fields were involved. In the absence of a rigorous derivation of a valid subtraction scheme for the calculation of the Euclidean action, it seems that one could engineer different schemes that gave different results, with no guide as to which result should be regarded as the correct one. 


\section{Viscosity/entropy ratio}

One of the motivations for this paper was to study the viscosity/entropy ratio in Horndeski gravity. Having obtained a formula for the entropy of the black holes, we are now in a position to proceed. To calculate the shear viscosity of the boundary field theory, we consider a transverse and traceless perturbation of the AdS planar black hole, namely

$$
d s^{2}=-f d t^{2}+\frac{d r^{2}}{f}+r^{2}\left(d x_{i} d x_{i}+2 \Psi(r, t) d x_{1} d x_{2}\right)
$$

where the background solution is given by $(2.11),(2.16)$ and (2.17). We find that the mode $\Psi(r, t)$ satisfies the linearised equation

$$
\begin{aligned}
& r(4 \kappa+\beta \gamma)\left(g^{2} r^{n-1}-\mu\right)^{2} \Psi^{\prime \prime}+(4 \kappa+\beta \gamma)\left(g^{2} r^{n-1}-\mu\right)\left(n g^{2} r^{n-1}-\mu\right) \Psi^{\prime} \\
&-r^{2 n-5}(4 \kappa-\beta \gamma) \ddot{\Psi}=0
\end{aligned}
$$

For an infalling wave which is purely ingoing at the horizon, the solution for a wave with low frequency $\omega$ is given by

$$
\begin{aligned}
\Psi & =e^{-\mathrm{i} \omega t} \psi(r), \quad \psi(r)=\exp \left(-\mathrm{i} \omega K \log \frac{f(r)}{g^{2} r^{2}}\right)+\mathcal{O}\left(\omega^{2}\right), \\
K & =\frac{1}{4 \pi T} \sqrt{\frac{4 \kappa-\beta \gamma}{4 \kappa+\beta \gamma}}
\end{aligned}
$$

Note that the constant parameter $K$ is determined by the horizon boundary condition. The overall integration constant is fixed so that $\Psi$ is unimodular asymptotically, as $r \rightarrow \infty$.

In order to study the boundary field theory using the AdS/CFT correspondence, we substitute the ansatz with the linearised perturbation into the action. The quadratic terms in the Lagrangian, after removing the second-derivative contributions using the GibbonsHawking term, can be written as

$$
\mathcal{L}_{2}=P_{1} \Psi^{\prime 2}+P_{2} \Psi \Psi^{\prime}+P_{3} \Psi^{2}+P_{4} \dot{\Psi}^{2}
$$

with

$$
\begin{aligned}
& P_{1}=-\frac{1}{8}(4 \kappa+\beta \gamma)\left(g^{2} r^{n-1}-\mu\right) r, \quad P_{2}=\frac{1}{2} g^{2} r^{n-1}[4 \kappa-(n-2) \beta \gamma]-\mu\left(2 \kappa-\frac{n-3}{4} \beta \gamma\right), \\
& P_{3}=\frac{n-1}{4} g^{2} r^{n-2}[4 \kappa-(n-2) \beta \gamma], \quad P_{4}=\frac{r^{2 n-5}(4 \kappa-\beta \gamma)}{8\left(g^{2} r^{n-1}-\mu\right)}
\end{aligned}
$$

Note that $P_{3}=\frac{1}{2} P_{2}^{\prime}$. We then find that the terms quadratic in $\Psi$ in the Lagrangian are given by

$$
\mathcal{L}_{2}=\frac{d}{d r}\left(P_{1} \Psi \Psi^{\prime}+\frac{1}{2} P_{2} \Psi^{2}\right)+\frac{d}{d t}\left(P_{4} \Psi \dot{\Psi}\right)-\Psi\left[P_{1} \Psi^{\prime \prime}+P_{1}^{\prime} \Psi^{\prime}+P_{4} \ddot{\Psi}\right] .
$$

The last term, enclosed in square brackets, vanishes by virtue of the linearised perturbation equation (4.2), and so the quadratic Lagrangian is a total derivative. The viscosity is 
determined from the $P_{1} \Psi \Psi^{\prime}$ term, following the procedure described in [6, 20]. Using this, we find that the viscosity is given by

$$
\eta=\frac{\kappa(n-1) \mu}{64 \pi^{2} T} \sqrt{1-\frac{\beta^{2} \gamma^{2}}{16 \kappa^{2}}}
$$

We have, for the planar black holes,

$$
\mu=g^{2} r_{0}^{n-1}, \quad T=\frac{(n-1) g^{2} r_{0}}{4 \pi},
$$

and the entropy that we derived in section 3.2 using the Wald formalism is given by

$$
S=\frac{1}{4} \kappa\left(1+\frac{\beta \gamma}{4 \kappa}\right) r_{0}^{n-2}
$$

We therefore find that the viscosity/entropy ratio is given by

$$
\frac{\eta}{S}=\frac{1}{4 \pi} \sqrt{\frac{4 \kappa-\beta \gamma}{4 \kappa+\beta \gamma}}
$$

for the Horndeski black holes. ${ }^{3}$ Note that $\kappa$ and $\beta$ are both positive. For reality, we must have

$$
-\frac{4 \kappa}{\beta}<\gamma<\frac{4 \kappa}{\beta}
$$

When $\beta=0$, which turns off the scalar field, the ratio goes back to the universal value of $1 /(4 \pi)$. When $\gamma>0$, the ratio is less than $1 /(4 \pi)$ and hence the bound is violated. For $\gamma<0$, the ratio is greater than $1 /(4 \pi)$.

Finally, we note that in terms of the original parameters of the theory (2.1), the viscosity/entropy ratio is given by

$$
\frac{\eta}{S}=\frac{1}{4 \pi} \sqrt{\frac{3 \alpha+\gamma \Lambda_{0}}{\alpha-\gamma \Lambda_{0}}} .
$$

Interestingly, the ratio is independent of the parameter $\kappa$.

\section{Conclusion}

Motivated by applications for the AdS/CFT correspondence, we studied the black holes in a theory of Einstein gravity coupled to a scalar field, including a non-minimal Horndeski term where the gradient of the scalar couples to the Einstein tensor. There are two types of static black holes in this Horndeski gravity. One of these is the usual Schwarzschild-AdS black hole, for which the scalar field is constant. Our focus is on the other non-trivial one-parameter family of static black holes, for which the scalar depends non-trivially on the radial coordinate. Although the scalar has a branch-cut singularity on the horizon, it is

\footnotetext{
${ }^{3}$ Intriguingly, although the ratio is calculated for the AdS planar black hole $(\epsilon=0)$, the same ratio $(4 \kappa-\beta \gamma) /(4 \kappa+\beta \gamma)$ appears in the sub-leading constant term in the large- $r$ expansion of $h=-g_{t t}$ given in (2.19), but only for the spherically-symmetric $(\epsilon=1)$ solutions (it vanishes for the $\epsilon=0$ solutions).
} 
axion-like and enters the theory only through a derivative. Furthermore, in an orthonormal frame, $\partial_{a} \chi$ is regular everywhere, both on and outside the horizon, and all invariants involving the scalar field are finite everywhere. We also demonstrated the uniqueness of these static black hole solutions in the theory.

We studied the thermodynamics of the black holes and found three surprises. The first is that the standard Wald entropy formula (1.5) does not give the complete expression for the entropy of these black holes. This can be attributed to the fact that the derivation of the Wald entropy (1.5) requires that the scalar be regular on the horizon. In fact, the branch cut singularity of the scalar on the horizon implies that there is an extra contribution to the entropy. We studied the Wald formalism in detail, and exhibited the new contribution explicitly. It turns out that the Wald identity (3.12) continues to hold for these black holes, and so does the first law of black hole thermodynamics. The entropy, however, is no longer given by (1.5), but can be determined from the implementation of the Wald procedure. We further established, using a simple construction of the Noether charge derivable from the scaling symmetry of the planar black holes, that the mass of the AdS planar black hole, as we derived from the Wald procedure, is indeed a conserved quantity.

The second surprise concerns the use of the quantum statistical relation $E-T S=$ $T I$ to calculate the thermodynamic parameters of the black hole solutions. In order to apply this method, it is necessary to calculate the Euclidean action $I$ of the black hole solution. The problem is that a direct integration of the Euclideanised action yields a result that diverges at the upper end of the radial integration, and so it is necessary to adopt some regularisation procedure. We tried to apply two different such procedures. The first involved subtracting the diverging contribution of a background where the mass is set to zero from the diverging contribution from the black hole with non-zero mass. The other procedure involved adding a boundary counterterm. The two methods gave the same results for the mass and the entropy, but these results differed from those that we obtained by using the Wald formalism. The origin of this mismatch is not clear to us; it may be related to intrinsic ambiguities in the subtraction schemes that we used in order to regularise the divergences. Such ambiguities are possibly more likely in a theory such as Horndeski gravity, with its somewhat unusual features, and so regularisation schemes for calculating the Euclidean action that usually work in less exacting situations may need to be scrutinised more carefully here.

The third surprise concerns the results in section 4 for the viscosity/entropy ratio. In wide classes of conventional theories with no higher-derivative terms in the Lagrangian, one finds a rather universal result that $\eta / S=1 /(4 \pi)$. Counter-examples to the universality of the ratio have been found, but for isotropic situations such as we have considered they are always associated with higher-derivative gravities, such as Gauss-Bonnet or more general Lovelock gravities. As far as we are aware, our findings for the black holes in the Horndeski theory we studied in this paper provide the first example of the violation of the $\eta / S=$ $1 /(4 \pi)$ result in a theory whose Lagrangian is at most linear in curvature tensor.

A word of caution about the use of the Wald formalism to calculate the entropy is perhaps appropriate here. If we consider Einstein-Maxwell theory as an example, the first law $d M=T d S+\Phi d Q$ for Reissner-Nordström black holes can be derived from the Wald 
formalism by calculating $\delta \mathcal{H}_{\infty}$ and $\delta \mathcal{H}_{+}$, and using the fact that $\delta \mathcal{H}_{\infty}=\delta \mathcal{H}_{+}$. The $\Phi d Q$ contribution can either enter in $\delta \mathcal{H}_{+}$alone, if one uses the gauge where the potential vanishes at infinity, or in $\delta \mathcal{H}_{\infty}$ alone, if one uses the gauge where the potential vanishes on the horizon, or else in both $\delta \mathcal{H}_{\infty}$ and $\delta \mathcal{H}_{+}$, if one uses some intermediate gauge where the potential vanishes neither at infinity nor on the horizon. In the first law, only the potential difference $\Phi \equiv \Phi_{+}-\Phi_{\infty}$ contributes. If the gauge where the potential vanishes on the horizon is chosen, then $\delta \mathcal{H}_{+}=T \delta S$ and so $\delta \mathcal{H}_{+} / T$ is an exact differential, which can be integrated to give the entropy, while $\delta \mathcal{H}_{\infty}=d M+\Phi_{\infty} d Q$, and is not exact. In the gauge where the potential instead vanishes at infinity, $\delta \mathcal{H}_{\infty}=d M$, which is an exact differential, while $\delta \mathcal{H}_{+}=T d S+\Phi_{+} d Q$, and so $\delta \mathcal{H}_{+} / T$ is not exact.

More complicated situations were encountered recently where asymptotically-AdS dyonically charged black holes were constructed in a four-dimensional gauged supergravity involving a scalar and a Maxwell field [47, 48]. It was found that $\delta \mathcal{H}_{\infty}$ was non-exact, and hence non-integrable, even when a gauge where the electric and magnetic potentials vanished at infinity was chosen, because of a varying contribution from the asymptotic coefficients in the large-distance expansion of the scalar field. The first law of black hole (thermo)dynamics, involving the scalar contribution, could nevertheless be derived using the strict Wald formalism [47]. The results were later generalised to black holes in general Einstein-scalar theories [39, 40], Einstein-Proca theories [41], and gravity extended with quadratic curvature invariants [43].

Analogous issues could in principle arise when considering $\delta \mathcal{H}_{+}$: it is commonly the case that $\delta \mathcal{H}_{+}$on the horizon can be expressed as $T \delta S$. In a theory such as Einstein-Maxwell, this is a gauge-dependent property as we discussed above, and in order to have $\delta \mathcal{H}_{+} / T$ be an exact differential in this case one would need to work in the gauge where the electric potential vanished on the horizon. In most theories that have been studied, the entropy is simply given by $S_{W}$ defined by the Wald entropy formula (1.5). The widespread validity of the Wald entropy formula is related to the fact that typically, matter fields vanish on the horizon of a black hole (and Maxwell potentials can be set to zero by means of appropriate gauge choices). In the Horndeski gravity considered in this paper, however, the axion-like scalar $\chi$ has an unusual behaviour near the horizon and near infinity, and indeed we have already seen that $\delta \mathcal{H}_{+} \neq T \delta S_{W}$. We nevertheless assumed that it was still the case that $\delta \mathcal{H}_{+}=T \delta S$, i.e. that $\delta \mathcal{H}_{+} / T$ could be integrated to define an entropy function. That $\delta \mathcal{H}_{+} / T$ is integrable is guaranteed in the one-parameter family of solutions considered in this paper, since all 1-forms in one dimension are exact. In a multiple-parameter black hole solution, however, there does not appear to be any guarantee, a priori, that $\delta \mathcal{H}_{+} / T$ must be a total differential in a theory such as Horndeski gravity. The non-integrability of the sort that occurs in $\delta \mathcal{H}_{\infty}$ in the dyonic asymptotically-AdS black holes we discussed above might also, in principle, occur for $\delta \mathcal{H}_{+} / T$ on the horizon, if not all the fields are strictly vanishing on the horizon. It would be interesting to study this further in more general solutions in theories such as Horndeski gravities.

The findings in this paper indicate that Horndeski gravity, and its black hole solutions in particular, deserve further investigation both in their own right, and also in the context of the AdS/CFT correspondence. 


\section{Acknowledgments}

We are grateful to Sera Cremonini for helpful discussions. H-S.L. is supported in part by NSFC grants 11305140, 11375153 and 11475148, SFZJED grant Y201329687 and CSC scholarship No. 201408330017. C.N.P. is supported in part by DOE grant DE-FG0213ER42020. The work of X-H.Feng and H.L. are supported in part by NSFC grants NO. 11175269, NO. 11475024 and NO. 11235003.

Open Access. This article is distributed under the terms of the Creative Commons Attribution License (CC-BY 4.0), which permits any use, distribution and reproduction in any medium, provided the original author(s) and source are credited.

\section{References}

[1] J.M. Maldacena, The large- $N$ limit of superconformal field theories and supergravity, Int. J. Theor. Phys. 38 (1999) 1113 [Adv. Theor. Math. Phys. 2 (1998) 231] [hep-th/9711200] [INSPIRE].

[2] S.S. Gubser, I.R. Klebanov and A.M. Polyakov, Gauge theory correlators from noncritical string theory, Phys. Lett. B 428 (1998) 105 [hep-th/9802109] [InSPIRE].

[3] E. Witten, Anti-de Sitter space and holography, Adv. Theor. Math. Phys. 2 (1998) 253 [hep-th/9802150] [INSPIRE].

[4] O. Aharony, S.S. Gubser, J.M. Maldacena, H. Ooguri and Y. Oz, Large- $N$ field theories, string theory and gravity, Phys. Rept. 323 (2000) 183 [hep-th/9905111] [INSPIRE].

[5] G. Policastro, D.T. Son and A.O. Starinets, The Shear viscosity of strongly coupled $N=4$ supersymmetric Yang-Mills plasma, Phys. Rev. Lett. 87 (2001) 081601 [hep-th/0104066] [INSPIRE].

[6] D.T. Son and A.O. Starinets, Minkowski space correlators in AdS/CFT correspondence: Recipe and applications, JHEP 09 (2002) 042 [hep-th/0205051] [INSPIRE].

[7] P. Kovtun, D.T. Son and A.O. Starinets, Holography and hydrodynamics: diffusion on stretched horizons, JHEP 10 (2003) 064 [hep-th/0309213] [INSPIRE].

[8] P. Kovtun, D.T. Son and A.O. Starinets, Viscosity in strongly interacting quantum field theories from black hole physics, Phys. Rev. Lett. 94 (2005) 111601 [hep-th/0405231] [INSPIRE].

[9] N. Iqbal and H. Liu, Universality of the hydrodynamic limit in AdS/CFT and the membrane paradigm, Phys. Rev. D 79 (2009) 025023 [arXiv:0809.3808] [inSPIRE].

[10] R.-G. Cai, Z.-Y. Nie and Y.-W. Sun, Shear viscosity from effective couplings of gravitons, Phys. Rev. D 78 (2008) 126007 [arXiv:0811.1665] [INSPIRE].

[11] R.-G. Cai, Z.-Y. Nie, N. Ohta and Y.-W. Sun, Shear viscosity from Gauss-Bonnet gravity with a dilaton coupling, Phys. Rev. D 79 (2009) 066004 [arXiv:0901.1421] [INSPIRE].

[12] R. Brustein, D. Gorbonos and M. Hadad, Wald's entropy is equal to a quarter of the horizon area in units of the effective gravitational coupling, Phys. Rev. D 79 (2009) 044025 [arXiv:0712.3206] [INSPIRE]. 
[13] H.-S. Liu, H. Lü and C.N. Pope, Generalized Smarr formula and the viscosity bound for Einstein-Maxwell-dilaton black holes, Phys. Rev. D 92 (2015) 064014 [arXiv:1507.02294] [INSPIRE].

[14] A. Buchel and J.T. Liu, Universality of the shear viscosity in supergravity, Phys. Rev. Lett. 93 (2004) 090602 [hep-th/0311175] [INSPIRE].

[15] A. Buchel, On universality of stress-energy tensor correlation functions in supergravity, Phys. Lett. B 609 (2005) 392 [hep-th/0408095] [INSPIRE].

[16] P. Benincasa, A. Buchel and R. Naryshkin, The shear viscosity of gauge theory plasma with chemical potentials, Phys. Lett. B 645 (2007) 309 [hep-th/0610145] [INSPIRE].

[17] K. Landsteiner and J. Mas, The shear viscosity of the non-commutative plasma, JHEP 07 (2007) 088 [arXiv:0706.0411] [INSPIRE].

[18] S. Cremonini, The shear viscosity to entropy ratio: a status report, Mod. Phys. Lett. B 25 (2011) 1867 [arXiv: 1108.0677] [INSPIRE].

[19] Y. Kats and P. Petrov, Effect of curvature squared corrections in AdS on the viscosity of the dual gauge theory, JHEP 01 (2009) 044 [arXiv: 0712.0743] [inSPIRE].

[20] M. Brigante, H. Liu, R.C. Myers, S. Shenker and S. Yaida, Viscosity bound violation in higher derivative gravity, Phys. Rev. D 77 (2008) 126006 [arXiv:0712.0805] [INSPIRE].

[21] M. Natsuume and M. Ohta, The shear viscosity of holographic superfluids, Prog. Theor. Phys. 124 (2010) 931 [arXiv: 1008.4142] [INSPIRE].

[22] J. Erdmenger, P. Kerner and H. Zeller, Non-universal shear viscosity from Einstein gravity, Phys. Lett. B 699 (2011) 301 [arXiv:1011.5912] [INSPIRE].

[23] O. Ovdat and A. Yarom, A modulated shear to entropy ratio, JHEP 11 (2014) 019 [arXiv: 1407.6372] [INSPIRE].

[24] X.-H. Ge, Y. Ling, C. Niu and S.-J. Sin, Holographic transports and stability in anisotropic linear axion model, arXiv:1412.8346 [INSPIRE].

[25] F.-W. Shu, The quantum viscosity bound in Lovelock gravity, Phys. Lett. B 685 (2010) 325 [arXiv: 0910.0607] [INSPIRE].

[26] J. de Boer, M. Kulaxizi and A. Parnachev, $A d S_{7} / C F T_{6}$, Gauss-Bonnet gravity and viscosity bound, JHEP 03 (2010) 087 [arXiv:0910.5347] [INSPIRE].

[27] X.O. Camanho and J.D. Edelstein, Causality constraints in AdS/CFT from conformal collider physics and Gauss-Bonnet gravity, JHEP 04 (2010) 007 [arXiv:0911.3160] [INSPIRE].

[28] C. Brans and R.H. Dicke, Mach's principle and a relativistic theory of gravitation, Phys. Rev. 124 (1961) 925 [INSPIRE].

[29] G.W. Horndeski, Second-order scalar-tensor field equations in a four-dimensional space, Int. J. Theor. Phys. 10 (1974) 363 [inSPIRE].

[30] A. Nicolis, R. Rattazzi and E. Trincherini, The galileon as a local modification of gravity, Phys. Rev. D 79 (2009) 064036 [arXiv:0811.2197] [INSPIRE].

[31] S.W. Hawking, Black hole explosions, Nature 248 (1974) 30 [INSPIRE].

[32] S.W. Hawking, Particle creation by black holes, Commun. Math. Phys. 43 (1975) 199 [Erratum ibid. 46 (1976) 206] [INSPIRE]. 
[33] R.M. Wald, Black hole entropy is the Noether charge, Phys. Rev. D 48 (1993) 3427 [gr-qc/9307038] [INSPIRE].

[34] V. Iyer and R.M. Wald, Some properties of Noether charge and a proposal for dynamical black hole entropy, Phys. Rev. D 50 (1994) 846 [gr-qc/9403028] [InSPIRE].

[35] A. Anabalon, A. Cisterna and J. Oliva, Asymptotically locally AdS and flat black holes in Horndeski theory, Phys. Rev. D 89 (2014) 084050 [arXiv: 1312.3597] [InSPIRE].

[36] M. Rinaldi, Black holes with non-minimal derivative coupling, Phys. Rev. D 86 (2012) 084048 [arXiv: 1208.0103] [INSPIRE].

[37] E. Babichev and C. Charmousis, Dressing a black hole with a time-dependent Galileon, JHEP 08 (2014) 106 [arXiv: 1312.3204] [INSPIRE].

[38] G.W. Gibbons and S.W. Hawking, Action integrals and partition functions in quantum gravity, Phys. Rev. D 15 (1977) 2752 [inSPIRE].

[39] H.-S. Liu and H. Lü, Scalar charges in asymptotic AdS geometries, Phys. Lett. B 730 (2014) 267 [arXiv: 1401.0010] [INSPIRE].

[40] H. Lü, C.N. Pope and Q. Wen, Thermodynamics of AdS black holes in Einstein-Scalar gravity, JHEP 03 (2015) 165 [arXiv:1408.1514] [INSPIRE].

[41] H.-S. Liu, H. Lü and C.N. Pope, Thermodynamics of Einstein-Proca AdS black holes, JHEP 06 (2014) 109 [arXiv: 1402.5153] [INSPIRE].

[42] Z.-Y. Fan and H. Lü, SU(2)-Colored (A)dS black holes in conformal gravity, JHEP 02 (2015) 013 [arXiv: 1411.5372] [INSPIRE].

[43] Z.-Y. Fan and H. Lü, Thermodynamical first laws of black holes in quadratically-extended gravities, Phys. Rev. D 91 (2015) 064009 [arXiv:1501.00006] [INSPIRE].

[44] H.-S. Liu and H. Lü, Thermodynamics of Lifshitz black holes, JHEP 12 (2014) 071 [arXiv: 1410.6181] [INSPIRE].

[45] W. Chen, H. Lü and C.N. Pope, Mass of rotating black holes in gauged supergravities, Phys. Rev. D 73 (2006) 104036 [hep-th/0510081] [INSPIRE].

[46] L.F. Abbott and S. Deser, Stability of gravity with a cosmological constant, Nucl. Phys. B 195 (1982) 76 [INSPIRE].

[47] H. Lü, Y. Pang and C.N. Pope, AdS dyonic black hole and its thermodynamics, JHEP 11 (2013) 033 [arXiv: 1307.6243] [INSPIRE].

[48] D.D.K. Chow and G. Compère, Dyonic AdS black holes in maximal gauged supergravity, Phys. Rev. D 89 (2014) 065003 [arXiv: 1311.1204] [INSPIRE]. 